\title{
Osteotomien nach in Achsenabweichung verheilten Frakturen im maxillofazialen Bereich
}

\author{
R. Gutwald, R. Schmelzeisen
}

\section{Zusammenfassung}

Sekundäre Rekonstruktionen von in Fehlstellung verheilten Frakturen des Unterkiefers und des Mittelgesichtes bedürfen einer exakten präoperativen Planung. Hierbei sind nicht nur die knöchernen Strukturen, sondern auch die umgebende Weichteilsituation $\mathrm{zu}$ beachten und in das Behandlungskonzept zu integrieren. Fortschritte in der orthopädischen Chirurgie (Dysgnathiechirurgie) sowie die Weiterentwicklung moderner Osteosyntheseverfahren haben wesentlichen Anteil an einer weitreichenden ästhetischen und funktionellen Reha- bilitation. Verbesserte bildgebende Verfahren sowie die Möglichkeiten der computerassistierten Chirurgie vereinfachen zudem Diagnostik, Planung und Durchführung der komplexen Eingriffe besonders im Mittelgesicht. Die konsequente Anwendung der modernen Diagnose- und Therapieverfahren sowie die interdisziplinäre Kooperation zwischen Neurochirurgie, Augenheilkunde, Hals-Nasen-Ohrenheilkunde, Kieferorthopädie und Mund-, Kiefer- und Gesichtschirurgie tragen heute ganz entscheidend zu einer signifikanten Verringerung der Mortalitäts- und Komplikationsraten bei.

\section{Einleitung}

In der maxillofazialen Traumatologie ist eine möglichst frühzeitige, definitive Wiederherstellung der knöchernen Strukturen einschließlich der Weichteilgewebe anzustreben. Durch ein meist gleichzeitig bestehendes Hirntrauma oder durch andere lebensbedrohliche Begleitverletzungen kann eine definitive knöcherne Rekonstruktion oft erst zu einem späteren Zeitpunkt erfolgen. Die primäre Behandlung beschränkt sich dann zunächst auf Stillung von Blutungen, Versorgung von Weichteilverletzungen und Anlegen von Schienenverbänden zur provisorischen Ruhigstellung vorhandener Frakturen.

Eine verzögerte knöcherne Primärversorgung sollte dann innerhalb von 10 Tagen bei Unterkieferfrakturen und innerhalb von 14 Tagen bei Mittelgesichtsfrakturen erfolgen. Ist in diesem Zeitraum weiterhin keine chirurgische Intervention mög-

OP-JOURNAL 2000; 16: 84-91

(C) Georg Thieme Verlag Stuttgart · New York lich, wird eine sekundäre knöcherne Rekonstruktion im Intervall notwendig. Dies gilt ebenso bei primär nicht erkannten oder in Fehlstellung verheilten Frakturen nach insuffizienter Primärversorgung, sowie Infektionen.

Vor einer Korrektur der Weichteile sollte stets die Rekonstruktion des knöchernen Lagers erfolgen. Eine simultane oder nachfolgende knöcherne Rekonstruktion kann in bestimmten Fällen, in denen starke Narbenzüge eine Resorption der knöchernen Rekonstruktion oder durch eine insuffiziente Weichteildecke Infektionen zu erwarten sind, indiziert sein.

Bei nicht versorgten oder instabilen Osteosynthesen ist die Infektions- und Pseudarthrosegefahr besonders im zahntragenden Anteil des Unter- und Oberkiefers deutlich erhöht. Aus diesen Gründen müssen Therapien im Sinne einer Infektionsbehandlung oder sekundäre Korrekturen häufig frühzeitig angegangen werden, um eine Verschlechterung der Knochen- und Weichteilsituation zu verhindern. Auf eine stabile Fixierung der Fragmente ist dann besonders zu achten. Die in der Regel bestehenden Knochendefekte werden durch autogene Transplantate bei reizlosem Zustand simultan oder bei bestehenden Infektionen im Intervall ausgeglichen.

\section{Unterkiefer}

Aufgrund verschiedener operativer Konzepte lassen sich posttraumatische Deformationen des Unterkiefers kausal in drei Kategorien unterteilen. Diese sind Folgen von Frakturen im Bereich des Kiefergelenkkopfes, des aufsteigenden Unterkieferastes oder des Unterkieferkörpers.

\section{Kiefergelenkkopf}

Knöcherne Verletzungen des Kiefergelenkkopfes, welche am häufigsten bei Kindern auftreten, können ohne Therapie zur Ankylose des Gelenkes mit resultierender Einschränkung der Unterkieferbeweglichkeit, insbesondere der Mundöffnung, führen. Die posttraumatische Funktionseinschränkung und die Therapie hängen vom Schweregrad der Ankylose und vom Alter des Patienten ab. Während bei leichten Formen einer Ankylose mit krankengymnastischen Übungsbehandlungen, kieferorthopädischen Funktionsgeräten oder arthroskopischen Eingriffen oft noch eine enorme Verbesserung der Mobilität des Unterkiefers erzielt werden kann, werden bei schweren Formen mit entsprechender Funktionseinschränkung offene operative Maßnahmen notwendig. Bei Kindern, die eine posttraumatische knöcherne oder schwere fibröse Ankylose aufweisen, sollte der ankylotische Bereich über einen extraoralen präauriculären Zugang reseziert und durch ein Transplantat ersetzt werden, welches noch ein spezifisches Wachstumspotential aufzeigt wie zum Beispiel ein Rippen-Knorpel-Transplantat. Bei Erwachsenen kann diese Therapie ebenfalls durchgeführt werden und sinnvoll sein. Hier kann aber schon die alleinige Durchtrennung der Ankylose mit folgender Interposition eines nicht resorbierbaren Materials zu 


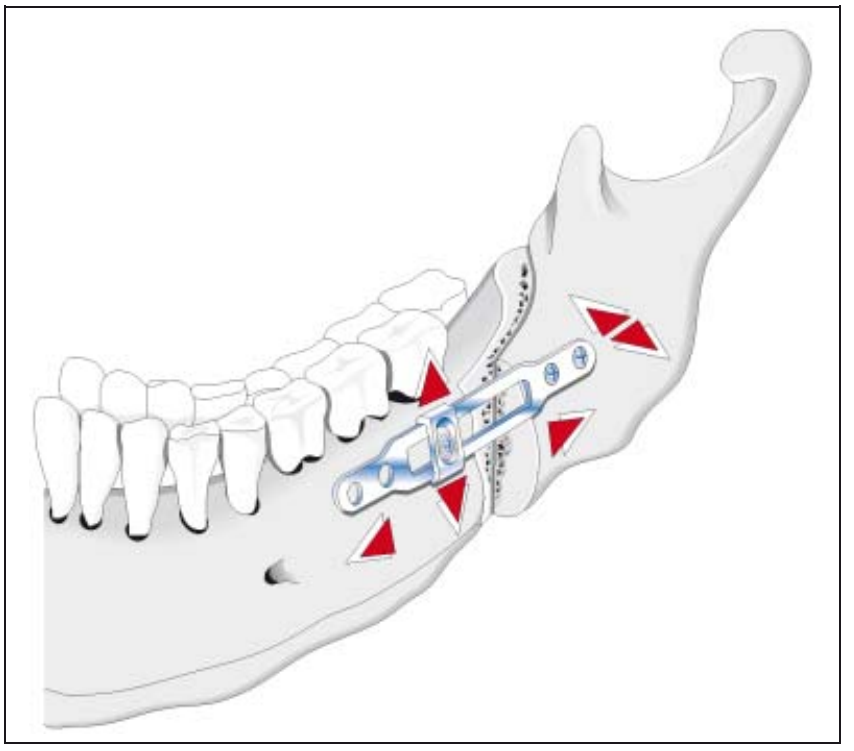

Abb.1 Sagittale Spaltung des Unterkiefers nach Obwegeser und Dal Pont. Horizontale Osteotomie an der Innenseite und vertikale Osteotomie an der Außenseite des Unterkiefers. Nach Verbindung der beiden Osteotomielinien an der Vorderkante des aufsteigenden Astes erfolgt die Spaltung in der Sagittalebene mit dem Meißel.

Fixation nach Unterkiefervorverlagerung mit einer SplitFix-Platte. Der temporär eingebrachte Schieber erlaubt Korrekturen in allen 3 Ebenen (Pfeile), bevor die anterioren Schrauben inseriert werden.

einer Verbesserung oder Wiederherstellung der Funktion führen. In beiden Fällen sind intensive postoperative Übungsbehandlungen notwendig, die eine gute Mitarbeit des Patienten voraussetzen.

\section{Aufsteigender Unterkieferast (Abb. 2-9)}

Bei der Fraktur des aufsteigenden Unterkieferastes, welcher sich vom Kieferwinkel bis zum Collum erstreckt, kommt es durch den Muskelzug der inserierenden Kaumuskulatur zu einer vertikalen Verkürzung des Unterkiefers mit Deviation zur frakturierten Seite. Folgen einer in dieser Fehlstellung verheilten Fraktur sind Okklusionsstörungen mit frontal offenem Biß und eine Verkürzung des lateralen Untergesichts. Bei den nicht seltenen bilateralen Frakturen im aufsteigenden Unterkieferast kann es zusätzlich zu einer erheblichen Dorsalverlagerung des Unterkiefers kommen.

Kleinere Fehlstellungen mit moderaten Okklusionsstörungen können unter Umständen kieferorthopädisch oder durch zahnärztliche prothetisch-konservierende Maßnahmen korrigiert werden. Dies gilt auch bei zahnlosen Patienten, die bei fehlenden Funktionsstörungen und unauffälliger Ästhetik durch eine prothetische Neuversorgung allein rehabilitiert werden können. Meist ist jedoch die Indi- kation zu einer operativen knöchernen Korrektur gegeben.

Hierbei wird nach Richtlinien der modernen orthopädischen Chirurgie (Dysgnathiechirurgie) skelettaler Kieferanomalien vorgegangen.

Nach Herstellung von Ober- und Unterkiefermodellen werden diese schädelund scharnierachsenbezogen in einem halbjustierbaren Planungsartikulator fixiert und eine Operationssimulation durchgeführt. Vorher ermittelte kephalometrische Daten und Weichteilanalysen werden dabei ebenfalls berücksichtigt. Nach der Modelloperation wird ein interokklusaler Splint hergestellt, der nach der Osteotomie und Verlagerung des proximalen Unterkiefers eingesetzt wird und somit die Position referenziert. Bei guter Primärverzahnung kann während der Okklusionseinstellung auch auf einen Splint verzichtet werden.

In der Regel wird die Osteotomie im Kieferwinkelbereich durchgeführt. Wir bevorzugen hierbei die durch einen intraoralen Zugang mögliche sagittale Spaltung des Unterkiefers nach Obwegeser und Dal Pont (Abb.1), da hierbei unter Schonung des $\mathrm{N}$. alveolaris inferior eine größtmögliche Kontaktfläche der osteotomierten Unterkieferfragmente gewährleistet wird.
Um die Position des gegenüberliegenden Kiefergelenkes nicht zu beeinflussen ist zur korrekten Einstellung der Okklusion eine bilaterale Osteotomie notwendig. In Ausnahmefällen kann auch eine unilaterale Osteotomie durchgeführt werden. Die osteosynthetische Fixierung erfolgt mit Miniplatten oder mit für die Dysgnathiechirurgie im Unterkiefer speziell entwickelten SplitFix-Platten. Diese Spezialplatten ermöglichen über einen Schieber intraoperative Korrekturen in allen drei Ebenen, ohne neue Schraubenlöcher zu bohren (Abb.1). Eine Fixierung ist auch mit drei bikortikalen Stellschrauben möglich.

\section{Unterkieferkörper (Abb.10-15)}

Frakturen im zahntragenden Abschnitt des Unterkiefers führen zu stufenförmigen Okklusionsstörungen und Asymmetrien der unteren Gesichtsregion. Im anterioren Unterkiefer kann es zudem zu einer transversalen Diskrepanz des Unterkieferbogens kommen.

Korrekturosteotomien werden weitgehend im Bereich der ehemaligen Fraktur durchgeführt.

Hierbei ist im seitlichen Unterkiefer besonders auf die Kontinuität des im Knochen verlaufenden $\mathrm{N}$. alveolaris inferior zu achten. Meist besteht schon durch das Trauma und die Fixierung in Fehlstellung eine irreversible Nervtraumatisierung mit An-, Hyp- oder Parästhesie der versorgenden Kinnregion.

Nach der Osteotomie und anatomischen Reposition bestehen zum größten Teil lokale Knochendefekte, die mit autogenem Knochen zum Beispiel aus dem Becken (Spongiosa, kortikospongiöser Span) aufgefüllt werden. Osteosynthestisch muss eine ausreichende Stabilität gewährleistet sein, um eine ungestörte Heilung zu erzielen.

Hierzu werden sogenannte Rekonstruktionsplatten verwendet, die mit mindestens vier Schrauben pro Fragmentseite fixiert werden sollten. Vorzugsweise verwenden wir dazu UniLOCK-Platten, bei denen durch eine Verriegelung der Schrauben in der Platte die Vorzüge des Fixateur-Prinzips ausgenutzt werden und ein Plattendruck auf den Knochen vermieden wird. Während im anterioren Unterkiefer ein intraoraler Zugang über das Vestibulum möglich ist, wird im posterioren Unterkiefer auch zur besseren Darstellung des Osteotomiebereiches ge- 

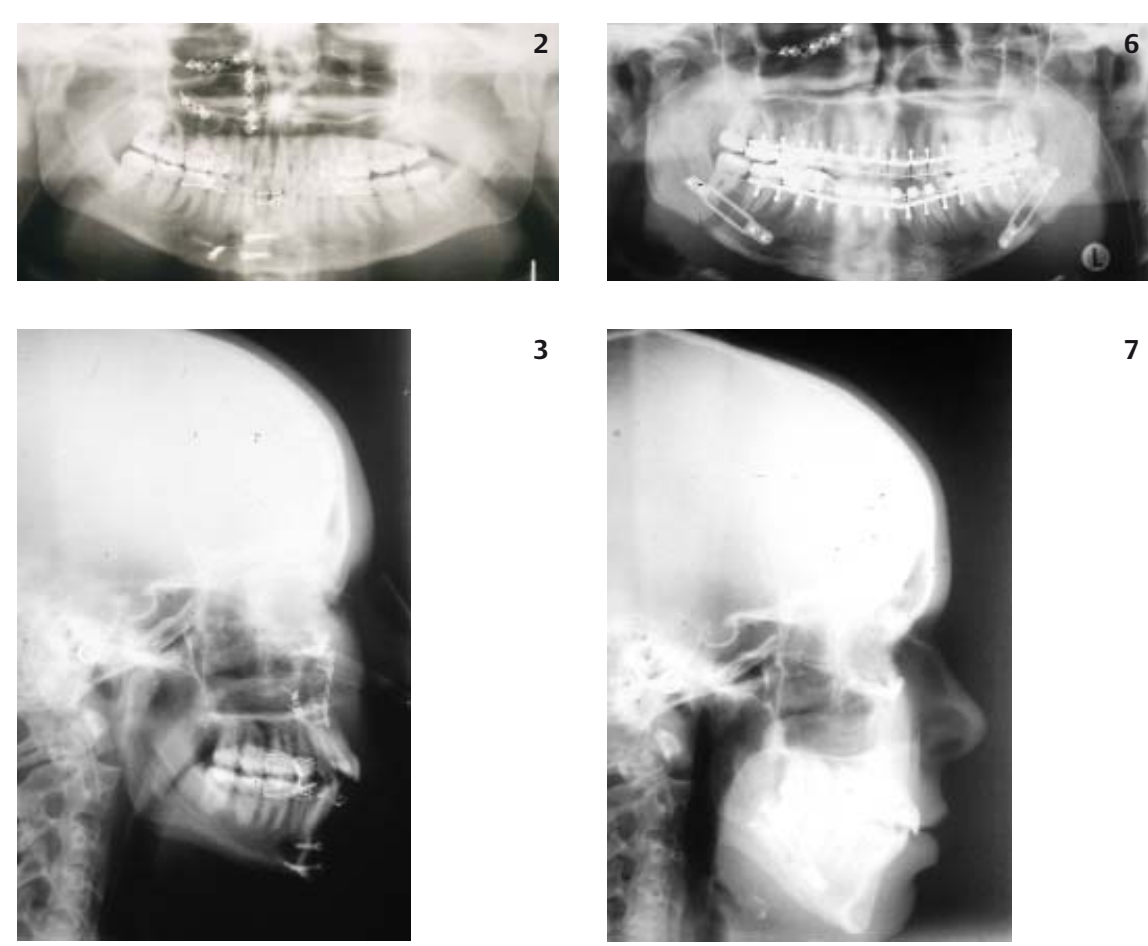

3
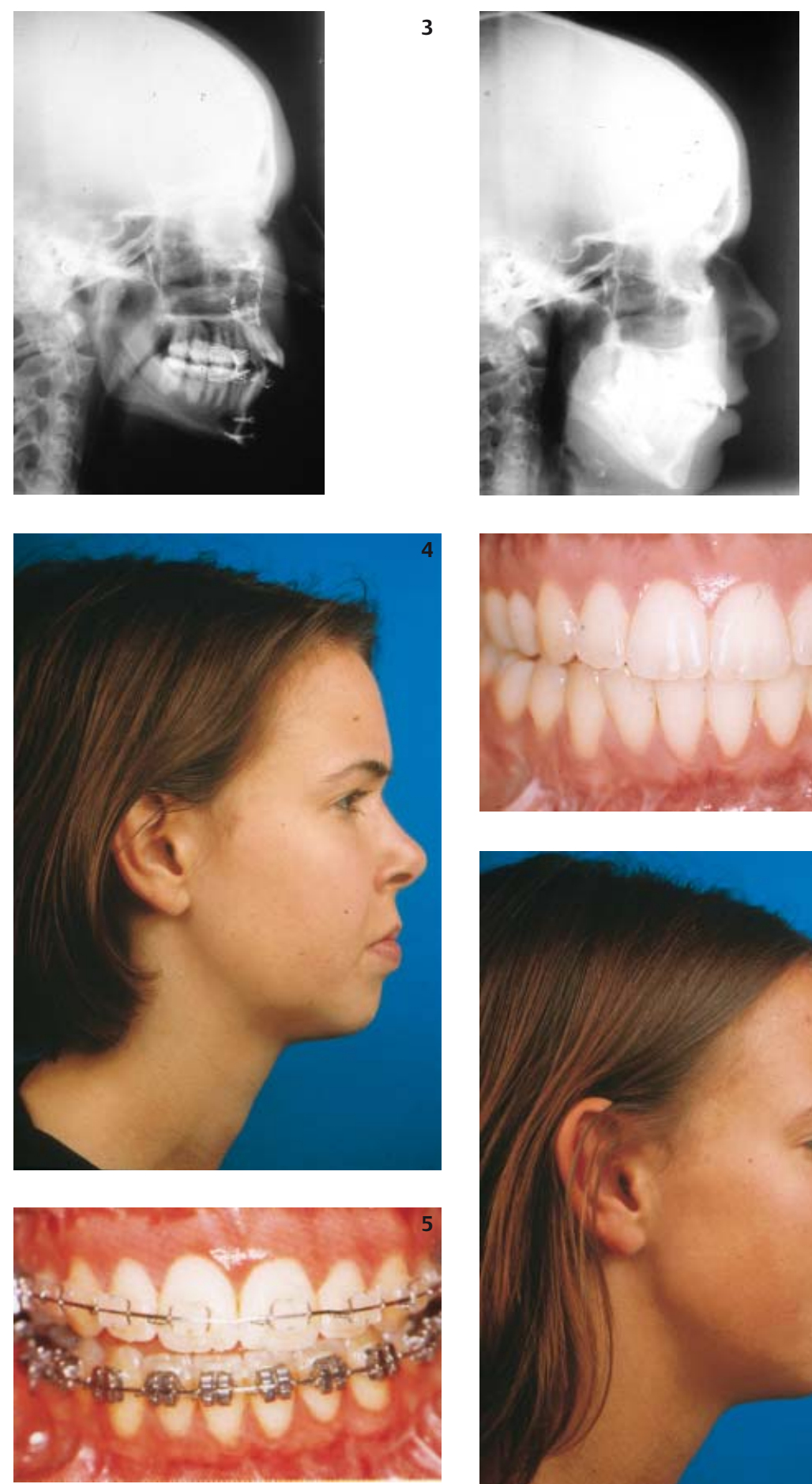

Abb. 2 Patientin mit paramedianer Unterkieferfraktur rechts, beidseitiger Collumfraktur und Jochbeinfraktur rechts. Postoperatives Röntgenbild (Orthopantomogramm) nach Primärversorgung. Die Paramedianfraktur wurde mit 3 Zugschrauben fixiert. Durch die nicht versorgte beidseitige Collumfraktur kam es zu einer Verkürzung des aufsteigenden Unterkieferastes auf beiden Seiten. Die eingebrachten Drahtligaturen waren für eine erforderliche intermaxilläre Immobilisation nicht ausreichend.

Abb. 3 Seitliches Fernröntgenbild der Patientin. Es sind deutlich die Dorsalverlagerung und vertikale Verkürzung des posterioren Unterkiefers erkennbar.

Abb. 4 Klinisches Profilbild der Patientin mit Rücklage und Verkürzung des lateralen Untergesichtes.

Abb. 5 Klinische Situation der Okklusion mit frontal offenem Biss besonders auf der rechten Seite. Die Zähne sind bereits mit kieferorthopädischen Drahtbogen versorgt, um die Zahnbogen vor der geplanten Korrekturoperation auszuformen.

Abb. 6 Postoperatives Röntgenbild nach Korrekturoperation durch bilaterale sagittale Spaltung und Ventralverlagerung des zahntragenden Unterkiefers. Osteosynthetische Fixierung beidseits mit SplitFix-Platten.

Abb.7 Seitliches Fernröntgenbild nach Korrekturoperation. Das Ausmaß der Unterkieferverlagerung nach ventral ist deutlich zu erkennen.

Abb. 8 Regelrechte Okklusion postoperativ mit vertikalem Überbiss der Frontzähne.

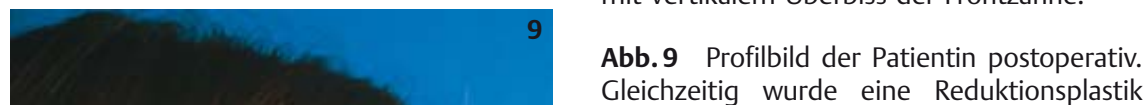
Gleichzeitig wurde eine Reduktionsplastik der Unterlippe durchgeführt.

wöhnlich ein extraoraler submandibulärer Zugang notwendig.

Vor der Entscheidung zur Osteotomie im ehemaligen Frakturbereich müssen unter Berücksichtigung aller Befunde (Röntgen, Modellanalyse, Ästhetik) alternative Möglichkeiten sorgfältig überprüft werden. So können moderate Okklusionsstörungen ohne Einschränkung der Ästhetik eventuell durch kieferorthopädische oder prothetisch-konservierende zahnärztliche Behandlungen allein ausgeglichen werden.

Knöcherne Fehlstellungen können bei bereits vorbestehenden skelettalen Kieferanomalien im Rahmen einer Dysgnathieoperation korrigiert werden. 


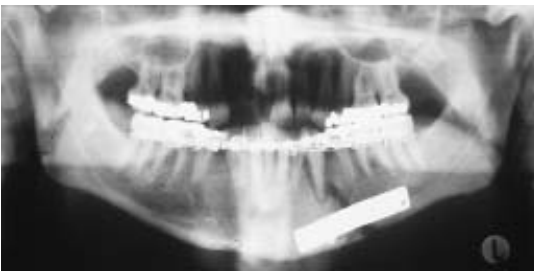

Abb.10 Röntgenbild eines Patienten nach Fraktur im horizontalen Unterkieferast links, welche mit einer alten DC-Stahlplatte am Unterkieferrand versorgt wurde. Durch Lockerung der Osteosynthese und eine postoperative Entzündung des Frakturbereiches ist es zu einer Pseudarthrose und Fehlstellung gekommen.

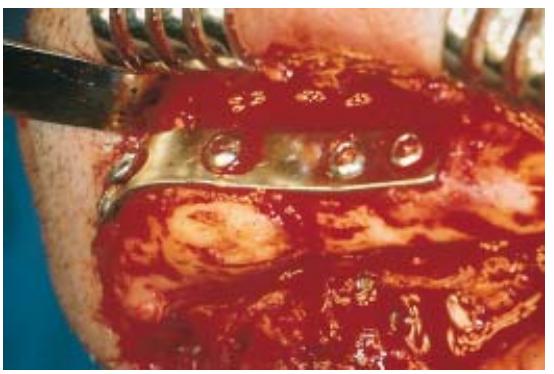

Abb.11 Intraoperatives Bild mit Darstellung der gelockerten Osteosynthesplatte und Pseudarthrose.

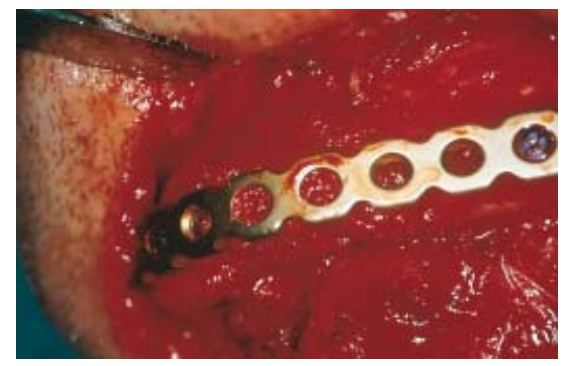

Abb.12 Intraoperatives Bild nach Resektion unter Schonung des N. alveolaris inferior und Auffüllung des Bereiches mit Spongiosachips aus der Beckenschaufel. Überbrückungsosteosynthese mit einer UniLOCK-Platte.

Neben einer kieferorthopädischen Vorbehandlung mit Ausformung der Zahnbogen ist hier die Auswirkung auf die Weichteilsituation zu berücksichtigen. Die Osteotomien werden nach präoperativer Planung wie bereits beschrieben vorzugsweise im Kieferwinkelbereich (bilaterale sagittale Spaltung des Unterkiefers nach Obwegeser und Dal Pont) vorgenommen und können bei Bedarf mit einer Osteotomie im Oberkiefer (Le Fort I Osteotomie) kombiniert werden. Eine kieferorthopädische Ausformung oder prothetisch-konservierende Rehabilitation kann unter Umständen auch erst

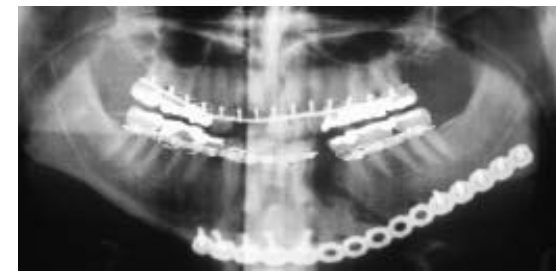

Abb.13 Postoperatives Röntgenbild nach Reposition in regelrechter Okklusion. Der Zahn 33 mußte intraoperativ entfernt werden. Fixation der UniLOCK-Platte mit jeweils 5 Schrauben anterior und posterior.

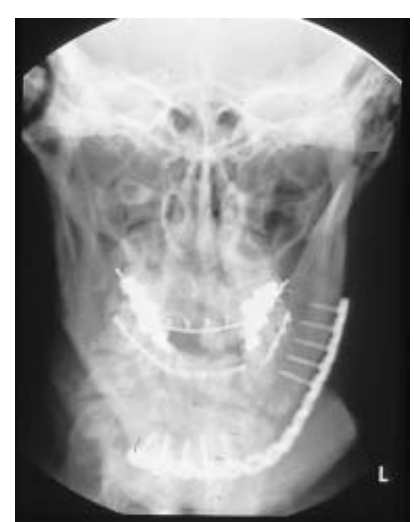

Abb.14 Postoperatives Röntgenbild (Schädel p.a.). Die Distanz der UniLOCK-Platte zum Knochen ist erkennbar.

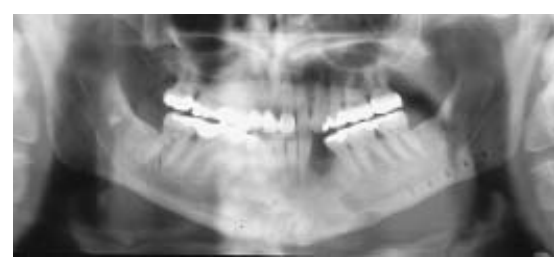

Abb.15 Röntgenbild nach Entfernung der UniLOCK-Platte im Abstand von einem halben Jahr zur Korrekturoperation. Gute knöcherne Überbrückung und Konsolidierung des resezierten Bereiches.

postoperativ erfolgen. In beiden Fällen ist eine gute und enge Zusammenarbeit der verschiedenen Fachdisziplinen Voraussetzung.

\section{Mittelgesicht}

Zu den häufigsten Deformationen nach nicht oder mangelhaft versorgten Mittelgesichtstraumen zählen Enophthalmus, vertikale Orbitadystopie, Jochbeinabflachung, Verbreiterung und Dorsalverlagerung des Mittelgesichtes zwischen den Jochbogen (Dish-Face), Verbreiterung und Impression des naso-orbito-ethmoi- dalen Komplexes, Telekanthus und Okklusionsstörungen.

Im Rahmen komplexer Mittelgesichtsrekonstruktionen ist daher eine enge interdisziplinäre Zusammenarbeit zwischen Neurochirurgen, Ophthalmologen, HalsNasen-Ohren-Ärzten, Kieferorthopäden und Mund-, Kiefer- und Gesichtschirurgen erforderlich, um ein für den Patienten ästhetisch und funktionell zufriedenstellendes Ergebnis zu erzielen.

Der limitierende Faktor bei sekundären Rekonstruktionen im Mittelgesicht ist die Weichteil- und Narbenkontraktur sowie die Adhärenz zu knöchernen Impressionen oder Defekten.

Bei Korrekturoperationen, wie sie früher üblich waren und lediglich mit Drahtosteosynthesen und Knochenaugmentationen durchgeführt wurden, kam es durch die Instabilität der Fixierung und der Weichteilkontraktion mit folgender Resorption der Knochentransplantate häufig zu einem Rezidiv mit erneutem Auftreten der Deformationen.

Die fortschrittlichen Osteosyntheseverfahren mit Mini- und Mikroplatten machen es heute möglich, eine adäquate Stabilisierung von Osteotomien und Transplantaten zu gewährleisten, um einen späteren Relaps der Strukturen zu vermeiden.

Kleinere Fehlstellungen, die vornehmlich die Ästhetik betreffen und keine funktionellen Defizite hervorrufen, können mit autogenen Knochentransplantaten (z.B. Tabula externa oder interna) kompensiert werden. Hierbei ist eine sichere Fixierung mit Osteosyntheschrauben oder Platten erforderlich. Im umgekehrten Fall kann eine Störung der Okklusion ohne Einschränkung der Ästhetik allein mit konservativen Maßnahmen durch kieferorthopädische oder prothetisch-konservierende Behandlungen ausgeglichen werden. Ist eine entsprechende Kompensation nicht möglich, wird eine knöcherne Rekonstruktion notwendig.

Korrekturosteotomien im Mittelgesicht werden in der Regel im Bereich der ehemaligen Frakturstellen durchgeführt. Diese liegen überwiegend in den Prädilektionsstellen der Le Fort I, II und III-Ebene (Abb. 16). Totalosteotomien in diesen Ebenen gelten auch als Standardverfahren in der modernen Dysgnathiechirurgie und Korrektur komplexer kraniofazialer Dysmorphien. 
Fehlende oder resezierte Knochenanteile werden mit geeigneten Knochentransplantaten ersetzt. Diese müssen wie die reponierten Knochenfragmente mit Mini- und Mikroplatten ausreichend fixiert werden. Ausnahmen bestehen in gering belasteten Regionen z.B. Orbitawände, wo auf eine osteosynthetische Fixierung bei kleineren Defekten verzichtet werden kann. Alternativ können auch resorbierbare Folien, speziell konturierte Platten oder Titangitter eingesetzt werden.

Bei vorbestehenden skelettalen Kieferanomalien ist eine gründliche präoperative interdisziplinäre Abklärung notwendig, ob eine Rekonstruktion der posttraumatischen Fehlstellungen simultan mit einer Korrektur einer eventuell bestehenden Kieferanomalie sinnvoll und durchführbar ist.

Infolgedessen kann eine Modifikation der Osteotomielinien zu den ehemaligen Frakturlinien, eine abweichende Repositionierung der knöchernen Fragmente oder eine Kombination mit einer Unterkieferosteotomie notwendig werden.

Eine adäquate Behandlung von Fehlstellungen im Mittelgesicht kann nur über eine übersichtliche Exposition aller ehemaligen Frakturbereiche und geplanten Osteotomiestellen erfolgen.

Im Bereich des Stirnbeins, der naso-orbito-ethmoidalen Region und des Jochbeins hat sich der Bügelschnitt (Koronarschnitt) etabliert, der eine weite Übersicht über die gesamte Frontalregion bis zum Os nasale und zu den Jochbogen erlaubt. Dadurch ist auch eine gleichzeitige Entnahme von Knochentransplantaten aus der Schädelkalotte (Tabula externa, Tabula interna) möglich. Durch infraorbitale, subziliare oder transkonjunktivale Inzisionen kann der Infraorbitalrand und der Orbitaboden dargestellt werden. Die laterale Oberlidinzision oder die laterale Augenbraueninzision erlauben die Darstellung des Stirnbeinpfeilers und der Sutura zygomaticofrontalis. Intraorale Schnittführungen im Vestibulum werden zur Exploration des zentralen Mittelgesichtes genutzt. Aber auch über bestehende Narben ist ein Zugang zum Osteotomie- oder ehemaligen Frakturbereich möglich.

Voraussetzung für die Planung und Durchführung von Korrekturosteotomien im Mittelgesicht ist eine eindeutige Identifizierung und kausale Zuordnung der Fehlstellungen.

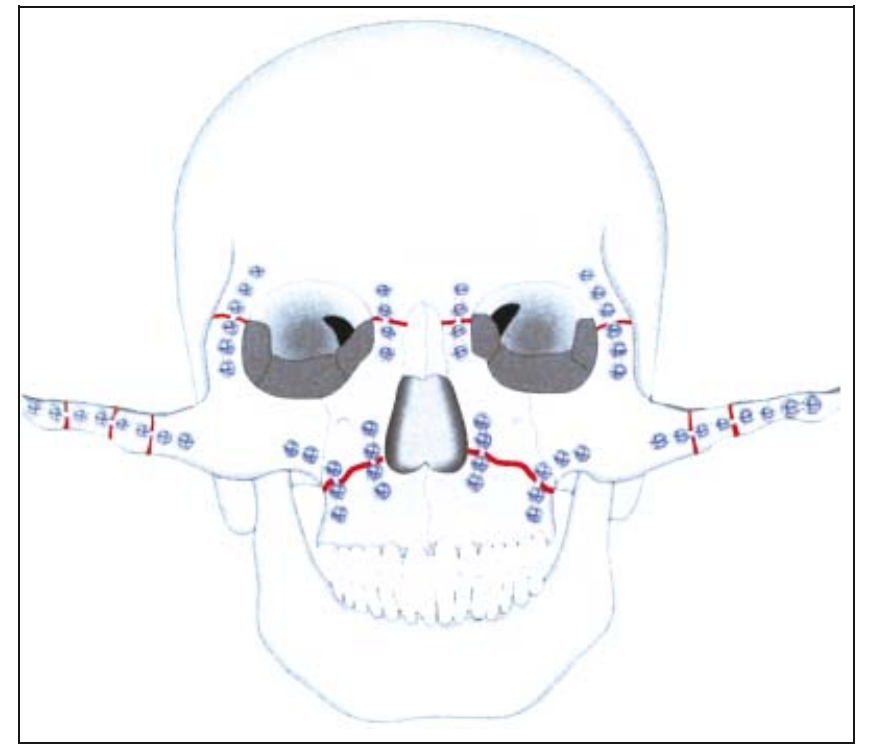

Abb. 16 Vereinfachte Darstellung der Osteotomie- und Fixierungsmöglichkeiten in der Le Fort I, II, III-Ebene.

Neben konventionellen Röntgenaufnahmen, Weichteilanalysen sowie Gipsmodellen des Unter- und Oberkiefers ist heute eine computertomographische Diagnostik mit dreidimensionaler Rekonstruktion fast unerläßlich.

Fortschritte in der komplexen Rekonstruktion sind zusätzlich von Seiten der computerassistierten Chirurgie gekommen. Diese hat im kraniofazialen Bereich andere Anforderungen als z.B. in der Neurochirurgie. Wir verwenden heute ein weiterentwickeltes multimodales System zur Planung, Simulation, und Navigations-Kontrolle bei komplexen Mittelgesichtsrekonstruktionen

(Abb.1723).

Voraussetzung für die computer-assistierte Chirurgie sind standardisierte Spiral-CT-Datensätze. Stereolithographiemodelle zur präoperativen Planung werden hierdurch vollständig ersetzt. Weitere Verbesserungen sind die präzise Quantifizierbarkeit von räumlichen Strukturen wie der Orbitadimensionen inklusive der Volumenveränderungen insbesondere bei komplexen posttraumatischen Enopthalmuskorrekturen.

Beim klinischen Einsatz im Operationssaal kann das Navigationssystem mittels Infrarot-Ortung über einen fest mit dem Patientenkopf verbundenen genormten Referenzierungsstern markierte Instrumente lokalisieren und somit jederzeit den virtuellen Patienten ohne jegliche Strahlenbelastung auf dem Computer in Form des CT-Datensatzes mit dem echten
Patienten auf dem Operationstisch korrelieren.

Damit diese Überlagerung zwischen dem echten und dem virtuellen Patienten deckungsgleich gelingt, bedarf es einer exakten Referenzierung. Daher werden vor der Datenakquirierung im CT-Marker am Patienten angebracht, die dann im Operationssaal zur Referenzierung dienen. Hierbei hat sich die Fixation der Marker an herausnehmbaren Dentalschienen im Oberkiefer bewährt, so dass nur noch bei zahnlosen Patienten unangenehme Fixationen über invasive Knochenmarker notwendig werden. Mit diesem noninvasiven patientenfreundlichen Eichverfahren sind nun viele Neuerungen in der komplexen rekonstruktiven Chirurgie umsetzbar.

Weiterhin kann bei nur einseitigen Deformationen mittels einer speziell entwickelten Software eine Spiegelung des Datensatzes durch Markierung einer virtuellen Ebene von der intakten auf die deformierte Seite innerhalb einer beliebigen kraniokaudalen Ausdehnung erfolgen. Ergebnis ist eine Idealrekonstruktion. Die Übereinstimmung der Position des osteotomierten Knochens oder von Transplantaten mit den idealisierten virtuellen Konturen kann dann während der Operation zu jeder Zeit navigiert werden. Dies erlaubt eine enorme Verbesserung im Hinblick auf Sicherheit und Vorhersagbarkeit des Rekonstruktionsergebnisses.

Bei der Diagnostik, Planung und operativen Rekonstruktion im Mittelgesicht ist 


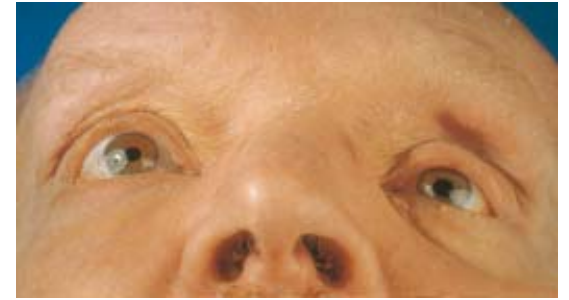

Abb.17 Patient mit vertikaler Orbitadystopie und Enophthalmus nach in Fehlstellung verheilter Jochbeinfraktur links.

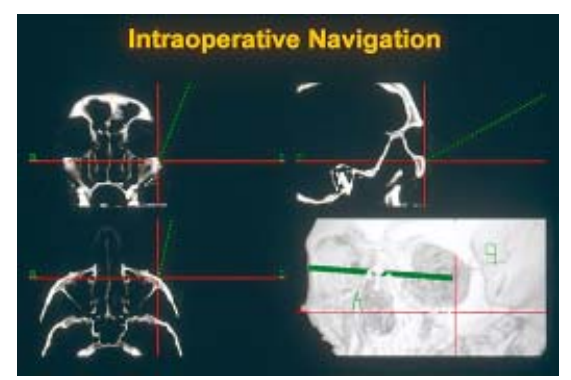

Abb.20 Intraoperative Navigation nach Osteotomie mit Kontrolle der Jochbeinprojektion durch einen Pointer (grüne gestrichelte Linie). Der grüne Balken entspricht der idealen anterioren Bulbusposition.

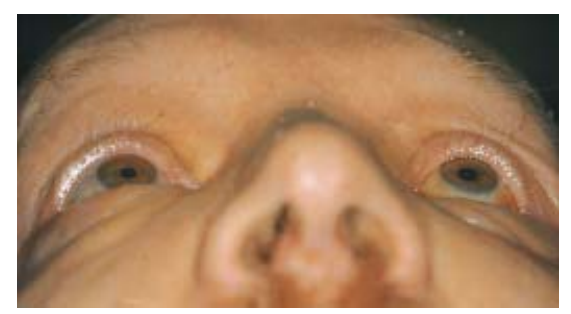

Abb. 23 Klinisches Bild des Patienten nach der komplexen Rekonstruktion.

auf spezielle Strukturkomplexe und deren anatomische und funktionelle Bedeutungen besonders zu achten.

\section{Zentrales Mittelgesicht}

Fehlstellungen nach Frakturen im Bereich der Le Fort I und II-Ebene äußern sich durch Abflachung und Elongation des Mittelgesichtes. Funktionell stehen Okklusionsstörungen mit in der Regel anterior offenem Biss im Vordergrund. Durch die posteriore Verlagerung wird klinisch der Eindruck einer mandibulären Hyperplasie mit umgekehrtem Überbiss erzeugt.

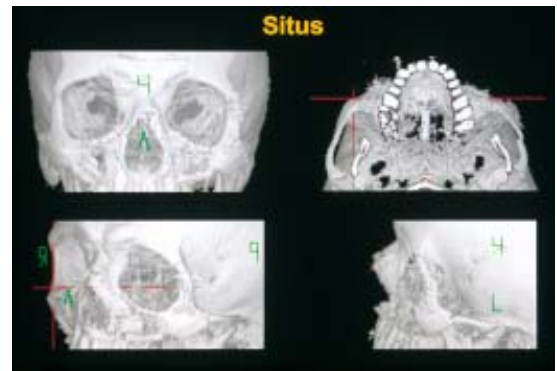

Abb.18 CT mit dreidimensionaler Rekonstruktion des Ausgangsbefundes.

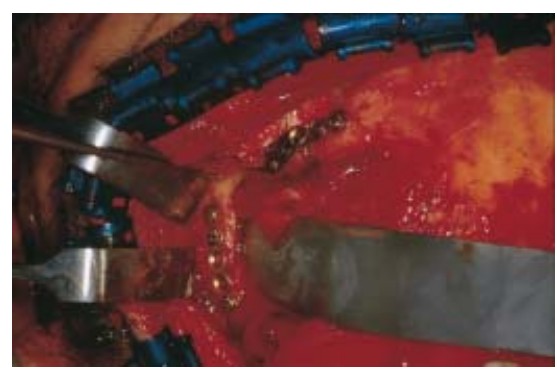

Abb. 21 Intraoperativer Situs über Bügelschnitt mit Darstellung der Miniplattenosteosynthese am Jochbogen und der lateralen Orbita.

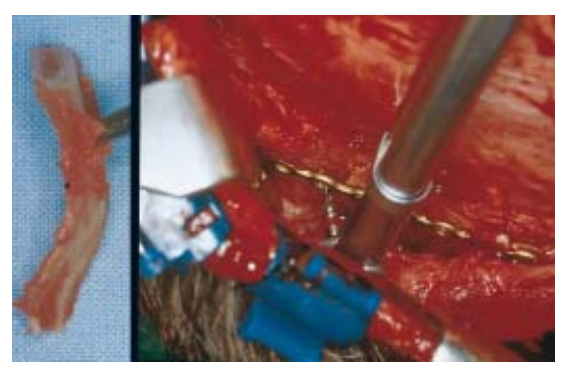

Abb. 24 Korrektur einer in Dislokation verheilten Jochbogenimpressionsfraktur durch Osteotomie des Jochbogens (links) und Fixation durch Miniplatte über einen Bügelschnitt (rechts).

Okklusionsstörungen nach isolierten Frakturen können weitgehend durch eine Osteotomie im Bereich der Le Fort I-Ebene korrigiert werden. Bei Bedarf sind Osteotomien einzelner zahntragender Oberkiefersegmente sowie eine sagittale Spaltung möglich. Lediglich bei stärkeren Abweichungen und ästhetischer Notwendigkeit wird eine Osteotomie in der Le Fort II-Ebene erforderlich. In allen Fällen wird wie in der Dysgnathiechirurgie eine präoperative Modelloperation durchgeführt und die Position des osteotomierten zahntragenden Knochensegmentes durch einen interokklusalen Splint referenziert.

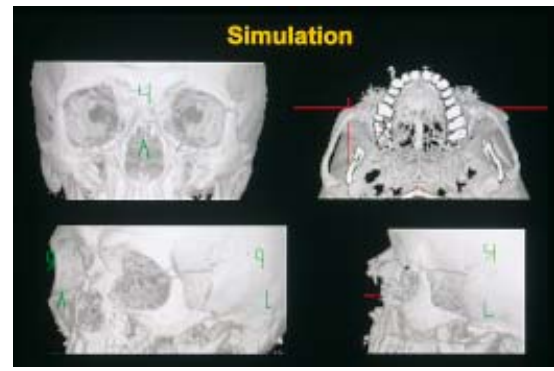

Abb.19 CT mit dreidimensionaler Rekonstruktion nach Spiegelung der intakten rechten auf die linke deformierte Seite.

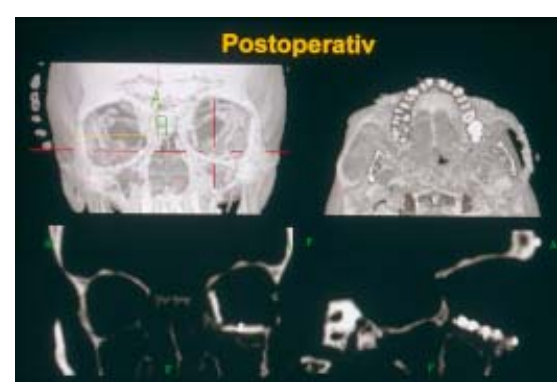

Abb. 22 Postoperatives Kontroll-CT nach Umstellungsosteotomie des Jochbeines links und Orbitarekonstruktion mit Tabula externa aus der Kalotte.

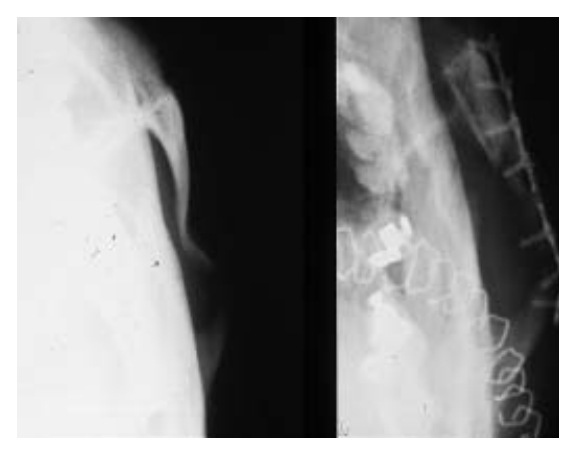

Abb. 25 Röntgenbilder (Henkeltopfaufnahmen) vor (links) und nach der Umstellungsosteotomie (rechts).

Bei Kombinationsfrakturen mit z. B. Le Fort III-Frakturen ist die Einstellung in der Le Fort I-Ebene das nivellierende Moment zwischen Mittelgesicht und Unterkiefer.

Dementsprechend werden die Positionierung und Fixierung dieses Segmentes mit Einstellung der Okklusion erst zum Abschluss komplexer Mittelgesichtsrekonstruktionen durchgeführt.

\section{Orbita}

Frakturen der orbitalen dünnen Wandstrukturen führen zu einer fehlenden $\mathrm{Ab}$ - 
stützung des intraorbitalen Weichgewebes. Durch die in der Regel nach peripher verlagerten Knochenanteile kommt es zu einer Vergrößerung des Orbitavolumens mit Enophthalmus. Begleitend kann eine vertikale Orbitadystopie vorliegen. Vereinzelte Knochendislokationen in den Orbitatrichter hinein führen dagegen zu einer Volumeneinengung mit Exophthalmus. Isolierte Wandfrakturen sind selten. Meist entstehen sie im Rahmen von zygomatico-orbitalen und naso-orbito-ethmoidalen Frakturen.

Bei der Rekonstruktion müssen die Defekte sicher aufgefunden und mit entsprechend konturierten autogenen Transplantaten ersetzt werden.

Ist eine gesicherte Abstützung noch vorhanden, wird eine osteosynthestische Stabilisierung nicht notwendig, da durch den Druck des intraorbitalen Weichgewebes die Transplantate in Position gehalten werden. Bei größeren Defekten, die mitunter alle vier Orbitawände betreffen können, ist eine Fixierung mit Mikroplatten und Schrauben unumgänglich. Die anatomische Wiederherstellung des Orbitatrichters lässt sich auch mit Hilfe von resorbierbaren Folien, speziell konturierten Orbitaplatten oder Titangittern, ermöglichen.

\section{Zygomatico-orbitale Region (Abb.17-25)}

Frakturen im Bereich des Jochbeinkomplexes mit Beteiligung der lateralen und inferioren Orbitawände führen in der Regel zu einer Abflachung und Verbreiterung des lateralen Mittelgesichtes. Meistens handelt es sich um Rotationsfehlstellungen des Jochbeins.

Bei der Rekonstruktion komplexer zygomatico-orbitaler als auch zentro-lateraler Mittelgesichttraumen spielt die Position der Jochbogen eine Schlüsselrolle.

Als Verbindung zwischen temporalem Anschluss nach posterior sowie Jochbein und Orbita nach anterior bestimmen sie die Position des lateralen und damit auch zentralen Mittelgesichtes. Daher muss zunächst die Form der Jochbogen rekonstruiert werden, um einen äußeren Rahmen zu bilden, der die vertikale und transversale Dimension des gesamten Mittelgesichtes definiert.

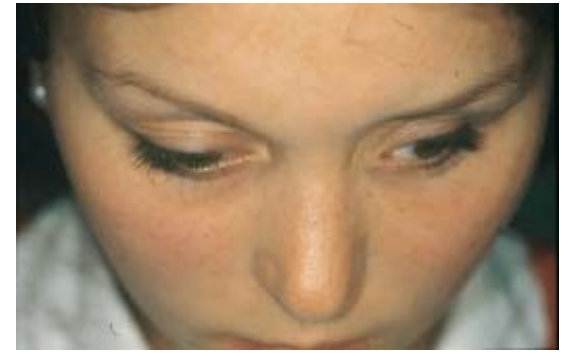

Abb. 26 Patientin mit linksseitigem Enophthalmus mit Doppelbildern sowie einem Telekanthus nach älterer Fraktur des naso-orbito-ethmoidalen Komplexes.

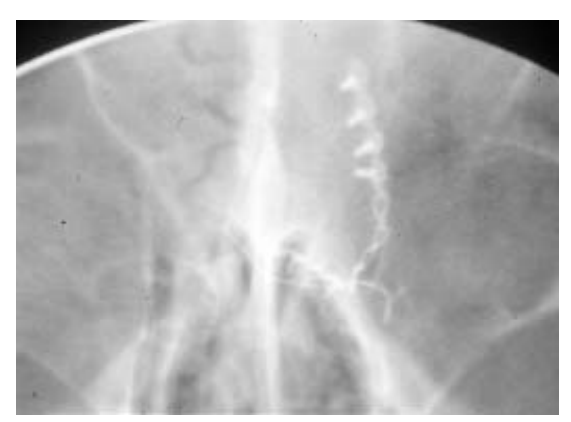

Abb. 27 Postoperatives Röntgenbild nach Osteotomie und Orbitarekonstruktion mit Tabula externa aus der Kalotte. Osteosynthese mit Microplatte und Reinsertion des medialen Lidbandes mit Draht.

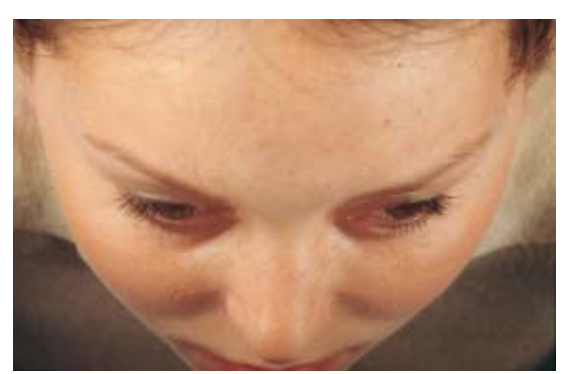

Abb. 28 Klinisches Bild der Patientin nach der Korrekturoperation.

\section{Naso-orbito-ethmoidale Region}

(Abb. 26-28)

Frakturen des naso-orbito-ethmoidalen Komplexes können isoliert oder im $\mathrm{Zu}$ sammenhang mit Frakturen in der Le Fort II und III-Ebene auftreten. Posttraumatische Deformationen entstehen durch Fehlstellungen des frontomaxillären Knochenpfeilers mit Verlagerung nach lateral, posterior und kaudal. Dabei wird das mediale Lidband, das meist am Knochen gestielt bleibt, mitverlagert. Insgesamt kommt es zu einer Einsenkung des interorbitalen Raumes mit Abflachung der Nasenwurzel und Verbreiterung des Augenlidabstandes (Telekan- thus). Moderate Fehlstellungen können mit einer Kombination aus Knochenkonturierung, Augmentation von Knochentransplantaten und transnasaler Kanthopexie ausgeglichen werden.

Bei ausgeprägten Dislokationen muss der gesamte Bereich osteotomiert und nach Reposition mit Mini- und Mikroplatten fixiert werden. Bei gleichzeitig bestehenden Frakturen in der Le Fort II oder IIIEbene sind die Osteotomielinien in das Gesamtkonzept zu integrieren. Kombinationen mit Knochentransplantaten sind häufig, da zum Versorgungszeitpunkt bereits eine Resorption der fragilen Knochenstücke stattgefunden hat.

Das mediale Lidbändchen sollte nach Osteotomie wieder mit Knochen replantiert werden. Dabei wird zusätzlich zur osteosynthetischen Fixation eine anteriore und insbesondere posterior des Ligamentes fixierte transnasale Drahtligatur zur Annäherung der beiden Knochenfragmente erforderlich. Wegen der bekannten Rückstelltendenz ist die Reposition mit einer gewissen Überkorrektur durchzuführen. Sollte das Lidband nicht mehr am Knochen adhärent oder dieser bereits resorbiert sein, wird eine Refixierung im Rahmen der Kanthopexie erforderlich.

\section{Schlussfolgerung}

Fortschritte in der orthopädischen Chirurgie (Dysgnathiechirurgie), Weiterentwicklung moderner Osteosyntheseverfahren, verbesserte bildgebende Verfahren und neue Möglichkeiten der computerassistierten Chirurgie haben zu einer enormen Verbesserung der Diagnostik, Planung und Therapie sekundärer Rekonstruktionen von posttraumatischen Fehlstellungen des Unterkiefers und des Mittelgesichtes geführt und damit zu einer weitreichenden ästhetischen und funktionellen Rehabilitation des Patienten.

Letztendlich können aber auch mit diesen Maßnahmen nicht alle Probleme und Spätfolgen eines komplexen kraniofazialen Traumas beseitigt werden, was die Bedeutung der korrekten Primärversorgung noch einmal unterstreicht. 


\section{Literatur}

${ }^{1}$ Gellrich N-C, Schramm A, Gutwald R, Schön $R$, Schmelzeisen R. Computer assisted planning and surgery in orbital reconstruction. In: Lemke HU, Vannier MW, Inamura K, Farman AG (Eds.): CARS'99 - Proceedings of the 13th Int. Congress and Exhibition, Elsevier, Amsterdam 1999; 1042

2 Greenberg A, Prein J. (Ed.). Craniomaxillofacial reconstructive and corrective bone surgery, Springer, New York 1998

3 Gruss, JS, Forrest CR. Craniofacial osteotomies and rigid fixation in the correction of posttraumatic craniofacial deformities. In: Bell WH (Ed.): Modern practice in orthognathic and reconstructive surgery, Saunders Company, Philadelphia 1992; $1012-1055$

4 Prein J. (Ed.). Manual of internal fixation in the cranio-facial skeleton, Springer, Berlin 1998
5 Schön R, Gellrich N-C, Gutwald R, Schmelzeisen R. Indication of selfdrilling screws in craniomaxillofacial surgery. Skull Base Surg 1999; 9 (Suppl. 2): 36

6 Schramm A, Gutwald R, Gellrich N-C, Thoma L, Schmelzeisen R. Reconstructive computer assisted surgery of deformities by mirroring data sets. Med Biol Eng Comp 1999; 37: 974-975

7 Spitzer WJ, Vanderborght G, Dumbach J. Surgical treatment of mandibular malposition after malunited condylar fractures in adults. J Cranio-Max-Fac Surg 1997; 25: 51 - 57

8 Taylor T D. Post-traumatic maxillomandibular deformities. In: Bell WH, (Ed.). Modern practice in orthognathic and reconstructive surgery, Saunders Company, Philadelphia 1992; 987-1011

9 Zachariades N, Mezetis M, Michelis A. Posttraumatic osteotomies of the jaws. Int J Oral Maxillofac Surg 1993; 22: 328-331
Dr. med. Dr. med. dent. R. Gutwald

Arzt für Mund-, Kiefer- und

Gesichtschirurgie

Prof. Dr. med. Dr. med. dent.

R. Schmelzeisen

Ärztlicher Direktor

Klinik und Poliklinik für Mund-, Kieferund Gesichtschirurgie der Albert-

Ludwigs-Universität Freiburg

Hugstetterstraße 55

79106 Freiburg 